\title{
Développement d'une méthodologie dans l'élaboration de lignes directrices pour de meilleures pratiques médicales
}

Recommandation $\operatorname{Rec}(2001) 13$ du Comité des Ministres aux Etats membres sur le développement d'une méthodologie dans l'élaboration de lignes directrices pour de meilleures pratiques médicales*

Le Comité des Ministres, en vertu de l'article 15.b du Statut du Conseil de l'Europe, considérant que le but du Conseil de l'Europe est de réaliser une union plus étroite entre ses membres et que ce but peut être poursuivi, entre autres, par l'adoption d'une action commune dans le domaine de la santé publique;

tenant compte des dispositions de la Convention de sauvegarde des Droits de l'Homme et des Libertés fondamentales et de la Charte sociale européenne;

rappelant que l'article 3 de la Convention sur les droits de l'homme et la biomédecine demande aux Parties contractantes de fournir «un accès équitable aux soins de santé de qualité appropriée», que l'article 4 de cette même convention exige que «toute intervention dans le domaine de la santé, y compris la recherche, doit être effectuée dans le respect des normes et obligations professionnelles», et que l'article 10 affirme que "toute personne a le droit de connaître toute information recueillie sur sa santé»;

rappelant les Recommandations du Comité des Ministres aux Etats membres $n^{\circ} \mathrm{R}$ (97) 5 relative à la protection des données médicales, $n^{\circ} \mathrm{R}$ (97) 17 sur le développement et la mise en œuvre des systèmes d'amélioration de la qualité des soins de santé, $n^{\circ} \mathrm{R}$ (99) 21 sur les critères de gestion des listes d'attente et des délais d'attente des soins de santé, ainsi que $\mathrm{n}^{\circ} \mathrm{R}(2000)$ 5 sur le développement de structures permettant la participation des citoyens et des patients au processus décisionnel concernant les soins de santé;

reconnaissant que les politiques en matière de santé et les systèmes de soins de santé doivent s'appuyer sur les meilleures données disponibles;

reconnaissant que les données médicales incluses dans les lignes directrices peuvent étayer les décisions nationales sur l'établissement de priorités en matière de besoins de santé, en tenant compte des questions éthiques, sociales et financières, des différences structurelles entre les systèmes de soins de santé et des variations des données épidémiologiques et de santé, mais ne devraient pas servir uniquement à des fins de compression des coûts et de rationnement;

reconnaissant le droit des patients et des citoyens à recevoir et à avoir facilement accès à une information pertinente au sujet de leur santé et des soins de santé les concernant, sous une forme et dans une langue compréhensibles pour eux;

considérant que les principes de meilleure pratique médicale s'appliquent de la même façon aux soins primaires, secondaires et tertiaires et à toutes les professions de santé, ainsi qu'aux activités de promotion de la santé, de prévention, de diagnostic, de traitement, de réadaptation et aux autres aspects des soins de santé;

reconnaissant que dans différents pays, des lignes directrices pour de meilleures pratiques médicales sont élaborées selon des modalités diverses, dans l'environnement complexe des systèmes de soins de santé, avec leurs aspects éthiques, économiques, sociaux, légaux ou autres;

considérant que la méthodologie pour l'élaboration et la mise en œuvre de lignes directrices dépasse les frontières nationales, et que l'interprétation et l'évaluation des données exigent des ressources et une expertise suffisantes, et que celles-ci devraient être menées en commun;

reconnaissant la nécessité de promouvoir une harmonisation des réglementations nationales et internationales concernant la recherche sur la qualité et la recherche clinique appliquée; reconnaissant que les lignes directrices ne sont qu'un moyen parmi d'autres d'améliorer la qualité et l'adéquation des services de santé et ne sauraient donc remplacer un jugement clinique sain, ni se substituer à la responsabilité professionnelle des prestataires non plus qu'aux préférences des patients;

considérant que les lignes directrices ont pour principal objectif de soutenir et de promouvoir une bonne pratique clinique, pour servir au mieux l'intérêt des patients, et devraient donc être considérées comme un guide 
pour l'action des pouvoirs publics, dont l'interprétation et le statut juridiques dépendent de la situation propre à chaque pays,

recommande aux gouvernements des Etats membres:

i. de développer un cadre général cohérent pour les politiques nationales qui:

- garantisse que les méthodes nationales d'élaboration et d'évaluation des lignes directrices pour de meilleures pratiques médicales soient conformes à l'état actuel des pratiques internationalement reconnues,

- garantisse que les décideurs, les professionnels de la santé, les citoyens et les patients reconnaissent l'intérêt d'utiliser les meilleures données disponibles pour fournir l'information nécessaire aux décisions médicales,

- soutienne l'élaboration, l'utilisation et l'actualisation régulière de lignes directrices sur la pratique clinique et les traitements médicaux s'appuyant sur des données scientifiques et pertinentes d'un point de vue national et local, en prenant pour objectif les questions importantes en matière de soins de santé,

- garantisse que les lignes directrices soient élaborées et mises en œuvre en tenant compte des aspects juridiques qui leur sont inhérents,

- garantisse que ces lignes directrices soient mises en œuvre de manière appropriée et que leurs effets sur les processus cliniques et leurs résultats ainsi que leurs conséquences juridiques concernant la position du malade et de celui qui dispense les soins de santé fassent l'objet d'un contrôle,

- facilite la disponibilité, dans une langue et sous une forme accessible, des lignes directrices et d'informations sur leur objectif, leur statut et leurs conséquences juridiques, de la documentation et des bases de données en matière de soins de santé et leur utilisation par les citoyens, les patients et les professionnels;

ii. de promouvoir le développement au niveau international de réseaux reliant les organisations, les répertoires et les autres organismes produisant une information médicale à base de données;

iii. de soutenir la diffusion active et ciblée de ces recommandations et de l'exposé des motifs, en accordant une attention particulière aux individus et aux organisations ayant un rôle à jouer dans les décisions en matière de soins de santé.

\section{Annexe à la Recommandation $\operatorname{Rec}(2001) 13$}

\section{Lignes directrices pour soutenir les soins de santé}

Les lignes directrices pour la pratique clinique ont pour objectif principal de soutenir et de promouvoir de bonnes pratiques cliniques.

Les lignes directrices sont élaborées et utilisées dans l'environnement complexe des systèmes de soins de santé, avec leurs aspects éthiques, économiques, légaux ou autres; ces différents aspects devraient être pris en compte dans chaque pays.

\section{Sélection des thèmes}

La sélection des thèmes à développer devrait viser à soutenir et à aider la prise de décision sur des questions importantes en matière de soins de santé.

La définition des thèmes de lignes directrices prioritaires peut reposer sur l'épidémiologie des problèmes de santé, les inégalités en matière de santé, les différences relatives à la fourniture et à la qualité des soins, le développement des nouvelles technologies ou d'autres facteurs faisant naître le besoin d'une information actualisée de haute qualité.

L'existence de lignes directrices à base de données actuellement disponibles devrait être prise en compte dans la définition des thèmes à développer en priorité.

\section{Elaboration des lignes directrices}

Les lignes directrices devraient être élaborées de manière systématique, indépendante et transparente par des groupes multiprofessionnels, en utilisant des critères de qualité adaptés.

La participation des usagers, au moyen d'un processus d'évaluation élargi et/ou de la mise à l'essai d'une version pilote, est nécessaire avant toute adoption de lignes directrices devant conduire à leur mise en œuvre.

Les lignes directrices provenant d'autres pays ou régions devraient faire l'objet d'une relecture et d'une révision, ou leur adaptation au nouveau contexte devrait être mise à l'épreuve.

\section{Diffusion des lignes directrices}

Le financement de la diffusion, de la mise en œuvre, de l'évaluation et de l'actualisation devrait être soigneusement envisagé au moment de la décision d'élaborer des lignes 
directrices. Les formes de financement peuvent varier. La source de financement doit être transparente.

Les lignes directrices devraient viser des publics multiples (professionnels de la santé, patients et décideurs) et être disponibles sous des formes adaptées à ces différentes catégories.

La diffusion des lignes directrices devrait être planifiée, active, durable et garantir leur accessibilité réelle.

Les répertoires de lignes directrices et les programmes d'élaboration de celles-ci facilitent l'accessibilité de nombreuses lignes directrices consacrées à des problèmes similaires et peuvent contribuer à la qualité des lignes directrices.

\section{Mise en œuvre des lignes directrices}

Pour assurer une mise en œuvre efficace des lignes directrices, une approche systématique en matière de gestion de la qualité des soins et de définition des responsabilités est essentielle.

Diverses stratégies de diffusion et de mise en œuvre des lignes directrices devraient être utilisées concurremment afin de leur assurer le maximum d'effet.

Des mécanismes professionnels, organisationnels, financiers et réglementaires d'incitation positive et négative devraient être envisagés parallèlement à d'autres moyens de faciliter l'utilisation des lignes directrices à l'échelon tant national que local (application sur mesure).

La mise en œuvre des lignes directrices devra privilégier l'intérêt du patient et respecter la responsabilité professionnelle et les droits des patients.

Les lignes directrices devraient devenir un élément essentiel de la formation initiale et de la formation clinique des professionnels de la santé, ainsi que du développement professionnel continu des équipes de soins.

\section{Evaluation des lignes directrices et de leurs effets}

Des instruments d'évaluation de la qualité des lignes directrices existantes devraient être utilisés pour déterminer les lignes directrices à mettre en œuvre.

Un contrôle soigneusement conçu des effets des lignes directrices est essentiel, et plus spécialement l'évaluation de leurs effets sur les résultats en matière de santé.

Les lignes directrices peuvent inclure une liste d'indicateurs fondamentaux à utiliser pour évaluer les résultats de leur mise en œuvre.

Un réseau de recherche coordonné au niveau international devrait étudier la méthodologie de l'évaluation des lignes directrices et du suivi de leur impact, y compris sur le processus d'apprentissage et les connaissances médicales des professionnels.

\section{Actualisation}

Le processus d'élaboration des lignes directrices devrait inclure des mesures claires pour l'actualisation des lignes directrices et préciser les responsabilités en ce domaine. 